\title{
Formal Systems as Physical Objects: A Physicalist Account of Mathematical Truth*
}

\author{
László E. Szabó \\ Theoretical Physics Research Group of the Hungarian Academy of Sciences \\ Department of History and Philosophy of Science \\ Eötvös University, Budapest \\ E-mail: leszabo@hps.elte.hu
}

\begin{abstract}
This paper is a brief formulation of a radical thesis. We start with the formalist doctrine that mathematical objects have no meanings; we have marks and rules governing how these marks can be combined. That's all. Then I go further by arguing that the signs of a formal system of mathematics should be considered as physical objects, and the formal operations as physical processes. The rules of the formal operations are or can be expressed in terms of the laws of physics governing these processes. In accordance with the physicalist understanding of mind, this is true even if the operations in question are executed in the head. A truth obtained through (mathematical) reasoning is, therefore, an observed outcome of a neuro-physiological (or other physical) experiment. Consequently, deduction is nothing but a particular case of induction.
\end{abstract}

\section{Introduction}

The central question of the philosophy of mathematics is what is mathematical truth, that is, what makes a mathematical proposition true.

Mathematical realism is the view that mathematical propositions are true insofar as they correspond with our physical environment. In other words, mathematics is an empirical science: mathematical propositions express the most general features of physical reality. Although it played an important role in the history of mathematical sciences, this view cannot be taken seriously in the time of modern mathematics. For there is no such direct correspondence between mathematical notions and the elements of physical reality. For example, nothing in the external world (outside mathematics) corresponds to the notion

*International Studies in the Philosophy of Science, forthcoming. 
of infinity. So, we reject the idea that "mathematics is an empirical science", as this thesis is usually understood, although, according to our final conclusion, we will see that it is an empirical science in another sense. As such, it does not express, however, the most general features of the physical world. On the contrary, it reflects some particular and not necessarily important features of it.

According to mathematical Platonism, substantive existence can be attributed to the classical concepts of mathematics, independently of whether or not anybody has these concepts in mind. A truth about a mathematical concept can be, like any other truth about any other existing thing, discovered. The particular way of discovery in which a true mathematical proposition can be obtained is the rational analysis of these concepts.

Intuitionists do not ascribe any existence to mathematical objects independent of their (evidently finite) construction by the basic intuition. Instead, they believe in the existence of "their god" (Curry, 1951, p. 6), Intuition, something which is a priori given to the universal human apprehension, something which, in this way, guarantees the objectivity and usefulness of mathematics.

Realists, Platonists and intuitionists jointly believe, however, that mathematical concepts and propositions have meanings, and when we formalize the language of mathematics, these meanings are meant to be reflected in a more precise and more concise form.

\section{The formalist thesis}

According to the formalist understanding of mathematics (at least, according to the radical version of formalism I am proposing here) the truth, on the contrary, is that a mathematical object has no meaning. "The formulas are not about anything; they are just strings of symbols" (Davis and Hersh, 1981, p. 319). Hilbert characterized mathematics as a game played according to certain simple rules with meaningless marks on paper (Bell, 1951, p. 38). That's all. Mathematics has nothing to do with the metaphysical concept of infinity. Mathematics does not produce and does not solve Zeno paradoxes. According to the formalist view (see Heyting, 1983, p. 71), one can write down a sign, say $\alpha$, and call it the cardinal number of the integers. After that, one can fix rules for its manip-

ulation. The whole finitist struggle is unnecessary. Such a sign as $10^{10^{10}}$ has no other meaning than as a figure on the paper with which we operate according to certain rules, just like any other symbols. Mathematical structures are totally indifferent to our intuition about space, time, probability or continuity. The words in a formal system have no meaning other than that which may be given to them by the axioms. As Hilbert - allegedly - expressed this idea in a famous aphorism about Euclidean geometry: "One must be able to say at all times - instead of points, straight lines, and planes-tables, chairs, and beer mugs." (Fang, 1970, p. 81)

Objecting to the formalist approach, many ask "How is it possible then that mathematics is applicable to the real world?" To be sure, mathematics is not "applicable" to the real world, but we can construct physical theories that do refer to the elements of reality. A physical theory $P$ is a formal system 
$L+$ a semantics $S$ pointing to the empirical world. In the construction of the formal system $L$ one can use previously prepared formal systems which come from mathematics and/or logic. For example, within the framework of a certain physical theory the physical space coordinates, as physical quantities, can be conveniently described in terms of Euclidean geometry. But this fact has nothing to do with the truth of a mathematical statement like $a^{2}+b^{2}=c^{2}$. The truth of such a statement means only that $a^{2}+b^{2}=c^{2}$ follows from the axioms, according to the derivation rules of that very formal system called Euclidean geometry.

It is an interesting philosophical question, of course, how a semantics $S$ works. But that problem is completely separate from the problems related to mathematics. This separation is obvious, if we take into account, for example, that a new empirical fact about physical space(time) can alter the corresponding physical theory (for example, we replace the whole Euclidean geometry with another one), while it leaves Euclidean geometry itself intact.

A sentence $A$ in physical theory $P$ can be true in two different senses:

$\operatorname{Truth}_{1}: \quad A$ is a theorem of $L$, that is, $L \vdash A$ (which is a mathematical truth within the formal system $L$, a fact ${ }^{1}$ of the formal system $L$ ).

Truth $_{2}$ : According to the semantics $S, A$ refers to an empirical fact (about the physical system described by $P$ ).

For example, "The electric field strength of a point charge is $\frac{k Q}{r^{2}}$ " is a theorem of Maxwell's electrodynamics - one can derive it from the Maxwell equations. (This is a fact of the formal system consisting of the symbols and the derivation rules.) On the other hand, according to the semantics relating the symbols of the Maxwell theory to the empirical terms, this sentence corresponds to an empirical fact (about the point charges). Truth ${ }_{1}$ and $\operatorname{Truth}_{2}$ are independent, in the sense that one does not imply the other. ${ }^{2}$

If mathematics is nothing but the science of formal systems consisting of meaningless marks and rules governing how these marks can be combined, then one can raise the question "What makes a system of marks and rules a formal system?" There is, however, no objective criterion for a system of marks and rules to be a formal system - and not every system of marks and rules counts as a formal system, relevant for mathematics. Some "structures" are traditionally regarded as "mathematical structures", and there are systems regarded as "formal/symbolic representations" of these "mathematical structures". Perhaps beauty and convenience are the two most important internal criteria mathematicians today have adopted to decide whether to study a structure as mathematical - as Mark Steiner (1998) sees it. Our concern here is not how this

\footnotetext{
${ }^{1}$ I do not have any scruple about using the word 'fact' here, since I actually mean 'empirical fact' about the formal system-as will be seen in section 4 .

${ }^{2}$ Moreover, assume that $\Gamma$ is a set of true 2 sentences in $L$, i.e., each sentence in $\Gamma$ refers to an empirical fact, and also assume that $\Gamma \vdash A$ in $L$. It does not automatically follow that $A$ is true 2 . If so, then it is new information about the empirical world, confirming the validity of the whole physical theory $P=L+S$ (including the rules of inference-of $L-$ in $P$ ).
} 
tradition has developed. Let us simply accept this tradition, and define a formal system as a system of marks and rules by which - according to the tradition-

a "mathematical structure is represented". For example, chess is a system of marks and rules, but it is not considered as representing a mathematical structure, so it is not a formal system. On the contrary, for example, the following simple system first defined and investigated by the logician Paul Lorenzen (see Podnieks, 1992, p. 28) is traditionally regarded as a formal system: The marks are strings consisting of letters $a$ and $b$. The axioms are the following:

$$
L= \begin{cases}a & \\ X \vdash X b & (\text { Rule 1) } \\ X \vdash a X a & \text { (Rule 2) }\end{cases}
$$

For example, the sequence of strings

$$
a \vdash a b \vdash a a b a \vdash a a b a b \vdash a a b a b b
$$

is a proof of the mathematical truth $a a b a b b$.

\section{The physicalist ontology of formal systems}

From the point of view of the original question of what makes a mathematical proposition true, the complete elimination of intuition, i.e. full reduction to a list of axioms and mechanical rules of inference, is possible. The work initiated by Frege, Russell, and Hilbert showed how this could be achieved even with the most complicated mathematical theories. No step of reasoning can be taken without a reference to an exactly formulated list of axioms and rules of inference. Even the most "self-evident" logical principles must be explicitly formulated in the list of axioms and rules. Thus, a precisely formalized mathematical derivation, making a mathematical proposition true, is like a "machinery of cogwheels", rather than the discovery of the "rational order in the world" by an "uncomputable consciousness" in its "clear and distinct intuitions".

The ontology of formal systems is crystal-clear: marks, say ink molecules diffused among paper molecules, more exactly, their interaction with the electromagnetic field illuminating the paper, or something like that. The rules according to which the marks are written on the paper form a strict mechanism which is, or easily can be, encoded in the regularities of real physical processes. Of course, a derivation on paper is rather similar to a production line in a factory employing low-paid workers: at certain points of the technological process human hands (and brains) transpose the workpiece from one conveyor belt to the other. This is, however, an unimportant technical problem. Since each step of manipulation is governed by strict rules, human beings can be replaced by trained animals, robots, etc. Also the marks can be of an entirely different nature, like, for instance, the cybernetic states of a computer, supervening on the underlying physical processes. For example, one can easily imagine a chip (a computer) designed to "realize" the formal system of Lorenzen described above: 
it can print strings consisting of letters $a$ and $b$. It starts by printing the string $a$. Then it works according to the mechanical rule: a string $X$ is followed by either $X b$ or $a X a$, and it prints all possible sequences of strings up to a certain length, then it prints out all possible sequences up to the double length, and so on. In a little while this machine will print out the proof (1) of the theorem $a a b a b b$.

Sometimes one executes simple formal derivations also in the head. ${ }^{3}$ However, from the point of view of the physicalist interpretation of mind this case of formal manipulation does not differ in principle from any other cases of derivation processes. If the signs and the rules of a formal system can be embodied in various physical states/processes, why not let them be embodied in the neurophysiological, biochemical, biophysical brain configurations-more exactly, in the physical processes of the human brain? If this is the case, that one of the paths - as some rationalists believe, the only path - to trustworthy knowledge, the deductive/logical thinking, can be construed as a mere complex of physical (brain) phenomena, without any reference to the notions of "meaning" and "intentionality", or the vague and untenable concept of the acausal "global" supervenience on the physical (cf. Chalmers, 1996, pp. 33-34), then this is, actually, a very strong argument for physicalism.

Moreover, there are derivations that are not 'surveyable' by the human mind - we cannot observe the whole derivation process, only the outcome of the process, the proved theorem. This is the case, for example, in the proof of the four-colour theorem (see Tymoczko, 1979), where certain steps of the proof are performed through very complex computer manipulations. Sometimes even the theorem obtained through the derivation process is not surveyable. It often happens, for example, that the result of a symbolic computer language manipulation is a formula printed on dozens of pages, completely incomprehensible to the human mind.

So, from certain point of view we must agree with David Deutsch, Artur Ekert, and Rossella Lupacchini:

Numbers, sets, groups and algebras have an autonomous reality quite independent of what the laws of physics decree, and the properties of these mathematical structures can be just as objective as Plato believed they were (and as Roger Penrose now advocates). But they are revealed to us only through the physical world. It is only physical objects, such as computers or human brains, that ever give us glimpses of the abstract world of mathematics. (Deutsch et al., 2000, p. 265)

On the one hand, Deutsch, Ekert, and Lupacchini rightly emphasize that mathematical truths are revealed to us only through real physical processes. On the other hand, they still maintain a Platonistic concept of truth in logic and

\footnotetext{
${ }^{3}$ Much more rarely than one would think. Even in the simplest cases, a proper formal derivation is much too complex to be executable in one's head.
} 
pure mathematics as independent of any contingent facts. The reason is the distinction they draw between knowledge and truth:

It seems that we have no choice but to recognize the dependence of our mathematical knowledge (though not, we stress, of mathematical truth itself) on physics, and that being so, it is time to abandon the classical view of computation as a purely logical notion independent of that of computation as a physical process. (Deutsch et al., 2000, p. 268)

Again, while one can accept the main message of this sentence, in the brackets Deutsch, Ekert, and Lupacchini take a step back towards the untenable Platonistic distinction between provability and (mathematical) truth. They are not radical enough as to recognize that the existence of a physical process of derivation that leads us to the knowledge of the truth of a mathematical proposition is nothing but the truth-condition of the mathematical proposition in question.

The same ambivalent views we find in Curry:

... although a formal system may be represented in various ways, yet the theorems derived according to the specifications of the primitive frame remain true without regard to changes in representation. There is, therefore, a sense in which the primitive frame defines a formal a system as a unique object of thought. This does not mean that there is a hypostatized entity called a formal system which exists independently of any representation. On the contrary, in order to think of a formal system at all we must think of it as represented somehow. But when we think of it as formal system we abstract from all properties peculiar to the representation. (Curry, 1951, p. 30)

What does such an "abstraction" actually mean? What do we obtain if we abstract from some unimportant, peculiar properties of a physical system $Z$ ? In accordance with what we said about the physical theories in the previous section, we obtain a theory $P=L+S$ about $Z$, that is, a formal system $L$ with a semantics $S$ relating the marks of the formal system to the (important) empirical facts of the physical system $Z$-where $L$ is a formal system in the head, or it is realized with paper and pen, etc. Now, the same holds if the physical system is a formal system (a "representation of a formal system", in Curry's terminology) $Z=L_{1}$ : through the abstraction we obtain a theory $L_{2}+S$ describing some important properties of the system $L_{1}$. That is, instead of an "abstract structure" we obtain another formal system $L_{2}$ "represented somehow"-in Curry's expression.

By the same token, one cannot obtain an "abstract structure" as an "equivalence class of isomorphic formal systems" or something like that, since in order to think of such things as "isomorphism", "equivalence", "equivalence class" at all we must think of them as living in a formal system "represented somehow".

The upshot of this is that "representation"--together with "translation", "isomorphism" and "understanding"- is a word to be avoided. As a complex of particular physical phenomena, a formal system is a metaphysically clear concept. 
There is no reason to suppose that it "represents" an "abstract mathematical structure"- there is no place where we could accommodate such an abstract structure, other than the Platonic realm or Popper's $n$th world or something like these. Since formal systems do not represent anything, it cannot be the case that different formal systems are different representations of some one common thing. Consequently, there is no "translation" between them. One formal system cannot "understand" the other.

Interaction is the proper term instead of representation, translation and understanding. That is what is going on in reality: a physical interaction between two formal systems as two particular physical systems. Wittgenstein would probably call the use of language the interaction of a brain with another formal system, first of all the interaction with another brain, which is usually realized through a third intermediate formal system. It is not our nominalism but rather an ontological clarity that keeps us from imagining "translation" or "understanding" of a "represented meaning", an "abstract mathematical structure", behind these interactions.

The whole language game is one complex of physical interaction processesinteractions between the builder's brain, the builder's body, the air (with the sound-wave patterns), the assistant's body, the assistant's brain, and the stone (cf. Wittgenstein 2002, p. 3). There is no "meaning" and no "intentionality" in this picture - just as there are no "abstract mathematical structures". These blue flowers are completely dissolved in physical reality. But we have ontological uniformity, instead. In this way, for example, a physical theory $P=L+S$ becomes intelligible for physics itself: not only is the formal system $L$ a physical system but also the semantics $S$ can be construed as a causal chain of physical interactions connecting the physical system $L$ with another physical system described by the theory $P$.

To sum up, a formal system is a physical system, the marks of the formal system are embodied in different phenomena related to the system and the derivation rules are embodied in the regularities of the system's behaviour. A mathematical derivation, making a mathematical proposition true, is nothing but a physical process going on in the formal system, and a theorem is the output of the process. To prove a theorem is nothing but to observe a derivation process in a formal system - that is, to observe a physical process in a physical system. That is all! In this physicalist ontological picture there are no "mathematical structures", as abstract thoughts, which are "represented" in the various formal systems.

Thus, physicalism-including the physicalist account of the mentalcompletes the formalist foundation of mathematics and removes the last residues of Platonism. The physicalist ontology of mathematical truth makes it completely pointless in mathematics to introduce a concept of truth different from that of being proved. Such a radical physicalist-formalist approach has interesting and important consequences in the philosophical analysis of Gödel's theorems and other foundational questions of mathematics, as I shall discuss elsewhere. In the last section of this paper we confine our attention to an important epistemological consequence of the physicalist ontology of formal systems. 


\section{Induction versus deduction}

It is a widespread opinion that one cannot justify a general statement about the world by induction. According to this opinion, deduction, contrary to induction, provides secure confidence because it is based on pure reasoning, without referring to empirical facts.

According to the key idea of rationalism, cognition is an independent source of trustworthy knowledge. Moreover, it is the only secure source of knowledge, the rationalists say, because cognition is the only source of necessary truth, while experience cannot deliver to us necessary truths, i. e., truths completely demonstrated by reason.

Let us leave aside the epistemological valuation of knowledge we obtain through inductive inference and consider in more detail the problem of deduction. The empiricist encounters difficulties in connection with the truths of formal logic and mathematics, as Ayer writes:

For whereas a scientific generalization is readily admitted to be fallible, the truths of mathematics and logic appear to everyone to be necessary and certain. But if empiricism is correct no proposition which has a factual content can be necessary or certain. Accordingly the empiricist must deal with the truths of logic and mathematics in one of the following ways: he must say either that they are not necessary truths, in which case he must account for the universal conviction that they are; or he must say that they have no factual content, and then he must explain how a proposition which is empty of all factual content can be true and useful and surprising. ...

If neither of these course proves satisfactory, we shall be obliged to give way to rationalism. We shall be obliged to admit that there are some truths about the world which we can know independently of experience; ... (Ayer, 1952, p. 72.)

According to the mathematical realist Mill, mathematical and logical truths are not certain and not necessary, since they are nothing but generalizations of our fundamental experiences about the physical world, and, as such, they are admitted to be fallible.

Logical empiricists, on the contrary, did not reject the necessity and certainty of mathematical and logical truths. According to their solution, analytical truths do not refer to the facts of reality. For we cannot obtain more information through deductive inference than that already contained in the premises. In other words, according to the logical empiricism, there are no synthetic $a$ priori statements.

Popper's falsification principle also accepts the necessity and certainty of mathematical and logical truths. This is the basis of the principal distinction between induction and deduction. Similarly, this principal distinction between the "trustworthy deductive inference" and the "always uncertain inductive generalization" is the fundamental tenet upon which the widely accepted hypothetico- 
deductive and Bayesian theories of science are built up, seemingly eliminating the problem of induction.

Now, from the standpoint of the physicalist ontology of formal systems, one can arrive at the following conclusion: mathematical and logical truths are not necessary and not certain, but they do have factual content referring to the real world.

For "deduction" is a concept which is meaningful only in a given formal system. On the other hand, as we have seen, a formal system is nothing but a physical system, and derivation is a physical process. The knowledge of a mathematical truth is the knowledge of a property of the formal system in question - the knowledge of a fact about the physical world. The formal system is that part of physical reality to which mathematical and logical truths refer.

It must be emphasized that this reference to the physical world is of a nature completely different from that assumed by Mill in his realist philosophy of mathematics. In the terminology we introduced in section 2 with respect to physical theories, the formal statements still do not have any reference to the real world in the sense of the truth-conditions of $\mathrm{Truth}_{2}$, since mathematics does not provide us with a semantics directed from the formal system to the outside world. When we are talking about the empirical character of mathematical truths, we are still talking about $\operatorname{Truth}_{1}$, namely we assert that even $\operatorname{Truth}_{1}$ is of empirical nature, the factual content of which is rooted in our experiences with respect to the formal system itself. Mathematics is, in this sense, empirical science.

The knowledge we obtain through a deductive inference is nothing but an empirical knowledge we obtain through the observation of the derivation process within the formal system in question. In other words, deduction is a particular case of induction. Consequently, the certainty of mathematics, that is the degree of certainty with which one can know the result of a deductive inference, is the same as the degree of certainty of our knowledge about the outcomes of any other physical processes.

For example, the reason why the truth of the height theorem is uncertain is not that our knowledge about the properties of "real triangles" is uncertain, as Mill takes it, but rather that our knowledge about the deductive (physical) process, the outcome of which is the height theorem, is uncertain, no matter how many times we repeat the observation of this process.

In order to explain the universal conviction that mathematical truths are necessary and certain, notice that there are many elements of our knowledge about the world which seem to be necessary and certain, although they are obtained from inductive generalization. If we need a shorter stick, we break a long one. We are "sure" about the outcome of such an operation: the result is a shorter stick. This regularity of the physical world is known to us from experiences. It can be known also to a chimp, from its own experiences obtained by trying to use a long stick. The certainty of this knowledge is, however, not less than the certainty of the inference from the Euclidean axioms to the height theorem.

Thus, mathematical truths are nothing but knowledge obtained through inductive generalization from experiences with respect to a particular physical 
system, the formal system itself. Reasoning is, if you like, a physical experiment. So, contrary to Leibniz's position that

There are ... two kinds of truths: those of reasoning and those of fact. The truths of reasoning are necessary and their opposite is impossible; the truths of fact are contingent and their opposites are possible. (Rescher, 1991, p. 21)

we must draw the following epistemological conclusion: The certainty available in inductive generalization is the best of all possible certainties!

\section{Acknowledgement}

The research was supported by the OTKA Foundation, No. T 037575 and No. T 032771 .

\section{References}

Ayer, A. J. (1952) Language, Truth and Logic (New York, Dover Publications).

Bell, E. T. (1951) Mathematics: Queen and Servant of Science (New York, McGraw-Hill Book Company).

Chalmers, D. J. (1996) The Conscious Mind (Oxford, Oxford University Press).

CurRY, H. B. (1951) Outlines of a Formalist Philosophy of Mathematics (Amsterdam, North-Holand).

DAvis, P.J. \& Hersh, R. (1981) The Mathematical Experience (Boston, Houghton Mifflin).

Deutsch, D., Ekert, A. \& Lupacchini, R. (2000) Machines, Logic and Quantum Physics, Bulletin of Symbolic Logic, 6, pp. 265-283.

FAnG, J. (1970) Hilbert: Towards a Philosophy of Modern Mathematics, (New York, Paideia Press)

Heyting, A. (1983) Disputation, in: P. Benacerraf \& H. Putnam (Eds) Philosophy of mathematics - Selected readings (Cambridge, Cambridge University Press), pp. 66-76.

Podnieks, K. M. (1992) Around Goedel's theorem (in Russian), 2nd edition, (Riga, Zinatne Publishers).

Steiner, M. (1998) The Applicability of Mathematics as a Philosophical Problem (Cambridge, Massachusetts: Harvard University Press).

Rescher, N. (1991) G.W. Leibniz's Monadology: An Edition for Students (Pittsburgh, University of Pittsburgh Press) 
Tymoczko, T. (1979) The Four-Color Problem and Its Philosophical Significance, Journal of Philosophy, 76, pp. 57-83.

Wittgenstein, L. (2002) Philosophical Investigations (Oxford, Blackwell). 